from recruitment visit (early-mid pregnancy) until child turns two years of age. Intention-to-treat and per-protocol analyses will be performed using general linear models to test the effects of interventions across three arms.

Results The trial was launched on 1 July 2020. As of 21 December 2020, a total of 60 participants were recruited with 2 of them withdrawn due to miscarriage. Currently, 14 participants have reached the postnatal stages. Participants' baseline socio-demographic characteristics were similar across three arms, with the mean age of 31 years old, and the mean gestation of 18.1 weeks, at the time of recruitment. In terms of ethnicity, majority of recruited patients were Chinese (71.7\%), followed by Malay (8.3\%), Others (11.7\%) and Indian (8.3\%). $90 \%$ of the participants were employed, and over $70 \%$ of the participants attained tertiary education qualifications.

Conclusions Despite recruitment delay of a few months due to COVID-19, the team was able to recruit 60 participants over 5 months with strict safe distancing measures. Multiple strategies have been established to facilitate the recruitment including extending publicity of the programme within and outside of KKH. The team will initiate preliminary data once 100 participants have been recruited. To engage existing participants, CRADLE will hold its first health education webinar in January 2021. This study may identify a sustainable strategy in the community by helping first-time parents to have a positive experience during the pregnancy, childbirth and parenthood, leading to enhanced PSE and health outcomes for both mother and child.

\section{PERCEPTION OF HONG KONG UNDERGRADUATES ON PERSONALIZED MEDICINE, PHARMACOGENOMICS AND GENETIC TESTING}

Nicholas Yan Chai Cheung, Christopher Chun Yu Mak, Jasmine Lee Fong Fung, Brian Hon Yin Chung. Hong Kong

10.1136/bmjpo-2021-RCPCH.133

Background The global development and advancement of genomic medicine in the recent decade has accelerated the implementation of Personalized Medicine (PM), Pharmacogenomics (PG) and Genetic Testing (GT) into clinical practice. The rapid emergence of diverse genetic services has marked the global transition to the genomic era. Our study aims at investigating the perception of Hong Kong (HK) undergraduates on PM, PG and GT.

Objectives To investigate the perception of Hong Kong undergraduates on Personalized Medicine, Pharmacogenomics and Genetic Testing

Methods By utilizing an online questionnaire based on a study published by Mahmutovic et al., this cross-sectional study was performed on 202 undergraduates of different study curriculum in the University of Hong Kong. Undergraduates' perception on three aspects were investigated general perception on PM, PG and GT; PM and PG education; and ethical, legal and social implications (ELSI) of GT. The primary outcome was the evaluation of undergraduates' perception on the above three aspects; and the secondary outcome was comparison of perception after stratification of undergraduates into medically and nonmedically-related curriculum. Fisher's exact test and Chi Square Test were performed for comparison of categorical responses, where the level of significance was set at p < 0.05 .

Results Our results showed that $80 \%$ of undergraduates valued $\mathrm{PM}$ as a promising healthcare model with $66 \%$ indicating awareness of personal genome testing companies. Despite this high awareness and interest, 60\% of undergraduates rated their genetic knowledge as 'School Biology' level or below and only 33\% would consider ordering a PG test for themselves. In contrast, $76 \%$ of undergraduates considered undergoing a genetic test, with $77 \%$ willing to have lifestyle modifications upon knowing their genetic risk of a disease. In terms of PM and PG education, slightly more than half of medically-related curriculum undergraduates perceived that their curriculum was well-designed for learning PG (52\%) and PG was important in their study (56\%); and only $16 \%$ of these undergraduates would consider embarking on future education on PM. Regarding ELSI, $75 \%$ of undergraduates were aware of ethical issues of GT in general and they were more concerned about 'Patient Privacy' (80\%) and 'Data Confidentiality' (68\%). Upon receiving an unfavorable result from genetic testing, majority of undergraduates perceived to feel 'helpless or pessimistic' (56\%), 'inadequate or different' (59\%) and 'disadvantaged at job seeking' (59\%), indifferent between medically and non-medically-related curriculum $(\mathrm{p}=0.24,1$, $0.24)$.

Conclusions Hong Kong undergraduates showed a high awareness of PM but in contrast there was insufficient knowledge and low interest in pursuing a career towards PM. They were generally aware of ethical issues of genetic testing and especially concerned about patient privacy and data confidentiality; and there appears to be a predominance of pessimistic views towards unfavourable genetic testing results. While this study may not be a representative of the general population, it calls for the attention to evaluate genomic education in Hong Kong.

This study was supported by Teaching Development Grant, The University of Hong Kong; and Common Core Curriculum, Scientific and Technological Literacy CCST9064 - The World Changed by DNA, The University of Hong Kong.

\section{NEONATAL THROMBOCYTOPENIA — INCIDENCE, RISK FACTORS, CAUSES AND OUTCOMES FOLLOWING PLATELET TRANSFUSIONS}

Wan Yi Yew, Varsha Atul Shah. Singapore

\subsection{6/bmjpo-2021-RCPCH.134}

Background Neonatal thrombocytopenia (NT) is defined as platelet counts less than $150,000 /$ microL, is most common haematological abnormality in the neonatal periods particularly in preterm infants and VLBW.

Objectives We evaluated the maternal-neonatal risk factors, causes, day of onset, duration of NT and the indications of platelet transfusions by means of a retrospective cohort study over a 3-years period.

Methods We conducted a retrospective analysis of prospectively collected data of all neonates born at Singapore General Hospital from year 2017 to 2019. Maternal characteristics like (age, number of pregnancies, medical conditions including maternal thrombocytopenia, pre-eclampsia, pregnancy induced hypertension, maternal age, intrauterine growth retardation, placental Doppler, mode of delivery and multiple births. 